in muscle carnitine levels and normal serum carnitine. Secondary carnitine deficiency is manifested by a decrease in levels of plasma or tissue carnitine, and is associated with genetically determined metabolic errors, acquired medical conditions, or iatrogenic factors. Metabolic errors involve fatty acid oxidation, B-oxidation cycle, aminoacidurias, and mitochondrial disorders. Acquired disorders include cirrhosis, malnutrition, vegetarian diet, celiac disease, extreme prematurity, AIDS, and Fanconi syndrome. Anticonvulsant treatment with valproate has been linked to some carnitine deficiencies. Several mechanisms are proposed for valproic acid-induced carnitine deficiency, some involving an underlying primary metabolic inborn error. The authors recommend prophylactic carnitine in all children under 2 years of age who are treated with valproate. Secondary carnitine deficiencies are managed by high carbohydrate, low fat frequent feedings, and vitamin/cofactor supplements (carnitine, glycine, and riboflavin). (Pons R, De Vivo DC. Primary and secondary carnitine deficiency syndromes. I Child Neurol November 1995;10(Suppl):2S8-2S24). (Respond: Dr Darryl C De Vivo, Neurological Institute, 710 West 168 Street, New York, NY 10032).

COMMENT. In the same issue, Coulter DL, from the Boston City Hospital, discusses the risk factors and treatment of carnitine deficiency in epilepsy. (I Child Neurol 1995;10(Suppl):2S32-2S39). Carnitine deficiency in epilepsy results from metabolic diseases, poor nutrition, and anticonvulsants, especially but not exclusively valproate. Carnitine supplements benefit high-risk, symptomatic patients and those with free carnitine deficiency, but not the low-risk, asymptomatic patients and those with normal carnitine levels.

\title{
PRIMARY LATERAL SCLEROSIS WITH GAZE PARALYSIS
}

Three children in a Jordanian family, with consanguinous parents, who met the Stark and Moersch (1945) criteria for the diagnosis of primary lateral sclerosis (PLS) are reported from the King Faisal Specialist Hospital and Research Centre, and King Khalid Eye Specialist Hospital, Riyadh, Saudi Arabia, and Northwestern University Medical School, Chicago, Illinois, USA. In addition they had a diffuse conjugate saccadic gaze paralysis, especially on down-gaze. A chronic progressive weakness beginning in late infancy, associated with spastic quadriplegia and pseudobulbar palsy, led to wheelchair dependence by adolescence and later loss of speech, while intellect was preserved. CT, MRI, EEG, EMG, NCS, and laboratory tests, including enzyme and amino acid assays, were normal. All patients had absent transcranial magnetic motor-evoked potentials in abductor pollicis and anterior tibial brevis muscles. Molecular testing, using DNA blood extracts, showed no linkage to chromosome 2q33 juvenile amyotrophic lateral sclerosis locus, the $8 \mathrm{q}$ recessive familial spastic paraplegia locus, or the 5 q13 spinal muscular atrophy locus. The clinical course and absence of specific neuropathological etiologies support the diagnosis of familial, autosomal recessive, primary lateral sclerosis. (Gascon GG, Siddique T et al. Familial childhood primary lateral sclerosis with associated gaze paralysis. Neuropediatrics 1995;26:313-319). (Respond: Dr Generoso G Gascon, Division of Pediatric Neurology, Rhode Island Hospital/Brown University, Physicians Office Building, Suite 438, 110 Lockwood Ave, Providence, RI 02903).

COMMENT. All three of these patients were referred with a diagnosis of cerebral palsy, despite the familial and progressive nature of the disorder. The authors cite only one other case of childhood primary lateral sclerosis in the literature. The present report is presented as the first of familial cases. Ford FR 
refers to cases of hereditary spastic paraplegia in children with degeneration confined to the pyramidal tracts. (Diseases of the Nervous System 4th ed, Springfield, CC Thomas, 1960, p 379).

\section{FUCOSIDOSIS WITH DYSTONIA}

A Canadian male child with fucosidosis and dystonia is reported from the Department of Biochemical Genetics, University of Western Ontario, London, Ontario, Canada. All milestones were delayed, he crawled at 14 months, walked by 18 months, and his developmental quotient at 27 months was 50 . At 46 months the quotient had dropped to 35. At 5 years, dystonic posturing of the left leg began, and 6 months later, he had episodes of choking, staring spells, and nocturnal apnea. At 7 years, he could not walk or talk, and the dystonia was bilateral. An angiokeratomatous rash became generalized. Cultured lymphoblasts showed absent a-fucosidase activity and protein. He was homozygous for the Q422X mutation. (Gordon BA et al. Fucosidosis with dystonia. Neuropediatrics 1995;26:325-327). (Respond: Dr BA Gordon, Department of Biochemical Genetics, CPRI, 600 Sanatorium Road, London, ON, Canada N6H 3W7).

COMMENT. Fucosidosis is a progressive neurodegenerative disease presenting in early childhood and manifested by loss of mental and motor function, with early hypotonia followed by increasing spasticity and seizures. Dystonia evident in this report had not previously been reported. Dysmorphic features and skeletal changes similar to those in mucopolysaccharidoses develop and include dwarfism, dysostosis multiplex, and visceromegaly. A skin rash occurs in those surviving childhood. The disease is caused by an autosomal recessive genetic deficiency of the lysosomal enzyme a-Lfucosidase. Seventy seven patient reports were reviewed in 1991. Menkes JH refers to two forms, type 1 without, and type 2 with angiokeratoma of the skin, particularly of the gingiva and genitalia. (Textbook of Child Neurology 3rd ed, Philadelphia, Lea \& Febiger, 19850.

\section{PURINE METABOLISM AND RETT SYNDROME}

Levels of purine and pyridine nucleotides and their metabolites were determined in erythrocytes and plasma of 31 Rett pattients and 17 age-matched controls at the Departments of Molecular Biology and Child Psychiatry, University of Siena, Children's Hospital, Siena, Italy. No difference was found in erythrocyte nucleotide concentrations, but plasma nicotinamide levels were significantly lower in Rett syndrome patients compared to controls. Erythrocyte activities of hypoxanthine phosphoribosyl transferase, adenine pbt and pbpp synthetase were also lower. The production rate of pyridine nucleotides from nicotinic acid by erythrocytes was increased in Rett patients. The significance of these metabolic changes was not determined. (Rocchigiani $\mathrm{M}$ et al. Purine and pyridine nucleotide metabolism in the erythrocytes of patients with Rett syndrome. Neuropediatrics 1995;26:288-292).

COMMENT. Despite frequent reports of various and diverse metabolic changes in patients with Rett syndrome, no consistent and diagnostic biochemical test has been identified for this disorder. The diagnosis is based on agreed clinical criteria. Alterations in nucleotide metabolism are an interesting addition to the search for a specific cause. 